\title{
Density-functional description of superconducting and magnetic proximity effects across a tunneling barrier
}

\author{
Jorge Quintanilla, ${ }^{1}$ Klaus Capelle, ${ }^{2}$ and Luiz N. Oliveira ${ }^{2}$ \\ ${ }^{1}$ ISIS Facility, STFC Rutherford Appleton Laboratory, Harwell Science and Innovation Centre, Didcot OX11 OQX, United Kingdom \\ ${ }^{2}$ Departamento de Física e Informática, Instituto de Física de São Carlos, Universidade de São Paulo, \\ Caixa Postal 369, 13560-970 São Carlos, São Paulo, Brazil
}

(Received 27 April 2008; revised manuscript received 23 October 2008; published 20 November 2008)

\begin{abstract}
A density-functional formalism for superconductivity and magnetism is presented. The resulting relations unify previously derived Kohn-Sham equations for superconductors and for noncollinear magnetism. The formalism, which discriminates Cooper-pair singlets from triplets, is applied to two quantum liquids coupled by tunneling through a barrier. An exact expression is derived, relating the eigenstates and eigenvalues of the Kohn-Sham equations, unperturbed by tunneling, on one side of the barrier to the proximity-induced ordering potential on the other.
\end{abstract}

DOI: 10.1103/PhysRevB.78.205426

PACS number(s): 71.15.Mb, 74.20.Rp, 75.70.Cn, 74.78.Fk

\section{INTRODUCTION}

Condensed-matter phenomena owe much of their variety to the multifarious properties of inhomogeneous electron liquids. Long-range order, gaps, screening, enhanced correlations, anomalies, resonances - a multiplicity of effects results from the diversity of chemical compositions and crystal structures. Not surprisingly, density-functional theory (DFT), a program dedicated to describing the effect of atomic-scale inhomogeneity on the electron liquid, has acquired mounting prominence. ${ }^{1,2}$ Its success in the description of properties associated with the microscopic inhomogeneities due to lattice potentials provides the foundation upon which $a b$ initio procedures are built. To describe ordered states, specialized formalisms comprising other order parameters along with the density have been proposed. ${ }^{3-7}$

Macroscopic inhomogeneities have received less attention from the DFT community. In particular a physical boundary separating distinct order parameters is a nesting ground for concepts and applications of which the discovery of giant magnetoresistances is the most persuasive example. ${ }^{8,9}$ More recently, advances in fabrication techniques and experimental probes (notably x-ray and neutron reflectometry) have revealed that the behavior at such boundaries can be quite unexpected. To give one example, novel magnetic order has been observed in the superconducting side of a ferromagnetsuperconductor interface (Ref. 10; see also Ref. 11 and references therein), yet one finds in the literature no DFT broadly applicable to junctions.

To fill this void, we present here a density-functional theory of superconducting and magnetic materials. A generalization of previously published theories for the individual orderings, ${ }^{3-7}$ the formalism is designed to describe longrange order with coexisting charge and magnetic and superconducting order parameters. Competition or coexistence of different forms of order is known to occur in bulk high-temperature, ${ }^{12}$ heavy fermion, ${ }^{13,14}$ and organic ${ }^{15}$ superconductors as well as manganites. ${ }^{16}$ Here we will focus on proximity effects in junctions between differently ordered quantum liquids. ${ }^{17}$

While studies of superconductivity in a specific material can be restricted to Cooper pairs of given spin, either singlets ${ }^{6,18}$ or triplets, ${ }^{18}$ the more general setups that we target require parallel treatment of the singlet pair, the triplet pair, and the magnetization densities. A triplet superconductor coupled to a noncollinear antiferromagnet is, e.g., well within the scope of our formalism.

As a general application, we consider two quantum liquids with different order parameters separated by a thin barrier that allows tunneling. Under these circumstances, we demonstrate that the Kohn-Sham (KS) equations yielding the ground-state energies and densities on one side of the barrier can be decoupled from the analogous equations for the energies and densities on the opposite side. In each decoupled KS Hamiltonian, an effective potential obtained from the solution of the unperturbed KS equations, i.e., the KS equations in the absence of tunneling, represents the opposite side. This potential gives mathematical substance, within DFT, to the proximity effect. In a normal metal-superconductor junction, the decoupled KS Hamiltonian adds correlation to the Bogolubov-de Gennes equations. ${ }^{19,20}$ In a normal metalantiferromagnet junction, it generates analogous equations describing the staggered proximity field induced on the nonmagnetic side.

Our presentation starts out with a cursory review of DFT for superconductivity and for magnetism. Section III presents the formalism for coexisting order parameters. The resulting KS equations are applied in Sec. IV to a barrier separating two quantum liquids, and the effective proximity potential is derived. In Sec. V the formalism is generalized to magnetic interfaces. Finally, Sec. VI lists our conclusions.

\section{DENSITY-FUNCTIONAL THEORY FOR ORDERED QUANTUM LIQUIDS}

Sections II A and II B briefly recapitulate the DFT approach to ordered quantum liquids. They highlight those aspects of the theory that will prove particularly important in our formulation and others that were brought to view in recent publications posterior to the original references.

\section{A. Density-functional theory for superconductors}

Superconductors still pose a challenge to electronicstructure theorists. Progress in that area had a late start. Long 
after band-structure calculations based on DFT had provided valuable information about such normal-state properties as Fermi-surface geometries, single-particle spectra, and electronic densities of states, well after DFT had yielded such lattice properties related to superconductivity as phonondispersion relations, the superconducting state still lay outside the realm of $a b$ initio electronic-structure calculations.

Model Hamiltonians had provided much of what was known about superconductivity. The reduced BCS Hamiltonian, ${ }^{21}$ the Hubbard Hamiltonian ${ }^{22}$ and its variations, and the Bogolubov-de Gennes mean-field equations ${ }^{19,20}$ are examples; none of which seemed adaptable to a densityfunctional formulation. In view of its reliance on empirical information on phonon spectra and Coulomb matrix elements, not even the detailed microscopic description of strong-coupling superconductivity in Eliashberg's theory could be seamed to ab initio DFT. ${ }^{23}$

To circumvent such difficulties, an alternative approach was proposed. ${ }^{6}$ Instead of functionals of the density, one now studied functionals of two variables: the normal density $n(\mathbf{r})=\left\langle\hat{\Psi}_{\uparrow}^{\dagger}(\mathbf{r}) \hat{\Psi}_{\downarrow}(\mathbf{r})\right\rangle$ and the superconducting order parameter $\chi\left(\mathbf{r}, \mathbf{r}^{\prime}\right)=\left\langle\hat{\Psi}_{\uparrow}(\mathbf{r}) \hat{\Psi}_{\downarrow}\left(\mathbf{r}^{\prime}\right)\right\rangle$. In the same way that $\rho$ is coupled to an electric potential, or the magnetization density of spinDFT (SDFT) is coupled to a magnetic field, the anomalous density $\chi$ was coupled to a pair potential. In the same way that the KS equations generalize the Hartree mean-field equations, extended KS equations were derived that generalize the mean-field Bogolubov-de Gennes equations.

Two are the potentials in these KS Bogolubov-de Gennes equations: an effective electric potential

$$
v_{s}(\mathbf{r})=v_{\text {ext }}(\mathbf{r})+v_{H}(\mathbf{r})+v_{\mathrm{xc}}(\mathbf{r}),
$$

and an effective pair potential

$$
\Delta\left(\mathbf{r}, \mathbf{r}^{\prime}\right)=\Delta_{\text {ext }}\left(\mathbf{r}, \mathbf{r}^{\prime}\right)+\Delta_{H}\left(\mathbf{r}, \mathbf{r}^{\prime}\right)+\Delta_{\mathrm{xc}}\left(\mathbf{r}, \mathbf{r}^{\prime}\right) .
$$

If the (microscopic) inhomogeneity is due exclusively to the lattice potential $v_{\text {ext }}$, only an infinitesimal external potential $\Delta_{\text {ext }}$ is needed to break the gauge symmetry that would otherwise annul the anomalous density in the self-consistent cycle. (As pointed out in Ref. 6, however, and further discussed in Sec. IV A, macroscopic inhomogeneities may generate noninfinitesimal external anomalous potentials.) While the Hartree potential $v_{H}$ tends to make the charge distribution uniform, the anomalous Hartree potential $\Delta_{H}$, the interaction of the anomalous density with its own pair potential, tends to enhance the superconducting order parameter. As usual, the exchange-correlation potential $v_{\mathrm{xc}}(\mathbf{r})=\delta E_{\mathrm{xc}}[n, \chi] / \delta n(\mathbf{r})$ is the derivative of the universal exchange-correlation functional of DFT with respect to the normal density. Similarly, $\Delta_{\mathrm{xc}}\left(\mathbf{r}, \mathbf{r}^{\prime}\right)=\delta E_{\mathrm{xc}}[n, \chi] / \delta \chi\left(\mathbf{r}, \mathbf{r}^{\prime}\right)$ represents the exchangecorrelation correction to the mean-field approximation. Among the generalizations of this formalism, we mention one that will assist our analysis: the extension to triplet superconductors. ${ }^{18}$

The exchange-correlation functional $E_{\mathrm{xc}}[n, \chi]$ is, of course, unknown, and the DFT program calls for firstprinciples approximations. That at least in the context of phonon-mediated superconductivity this program can be fol- lowed to its end was demonstrated by Gross and co-workers, ${ }^{24-30}$ who constructed a functional with no adjustable parameters and applied it to a number of materials. We note in passing that their breakthrough has led to the first truly microscopic theory of conventional superconductivity.

\section{B. Density functional for magnetic systems}

The fundamental variables in the standard formulation of DFT, collinear SDFT, are the spin-resolved densities $n_{\uparrow}(\mathbf{r})$ and $n_{\downarrow}(\mathbf{r})$. From these, the charge density $n(\mathbf{r})=n_{\uparrow}(\mathbf{r})$ $+n_{\downarrow}(\mathbf{r})$ and the magnetization $m_{z}(\mathbf{r})=\mu_{0}\left[n_{\uparrow}(\mathbf{r})-n_{\downarrow}(\mathbf{r})\right]$ are promptly recovered ( $\mu_{0}$ is the Bohr magneton). We concentrate our discussion on two features of magnetic systems that are foreign to SDFT in the local spin-density approximation: noncollinearity and nonlocality.

To describe noncollinear magnetic structures, one may substitute the magnetization vector $\boldsymbol{m}(\mathbf{r})$ for its $z$ component. Elegant extensions of noncollinear SDFT and successful implementations have been cast in this forge. ${ }^{31-34}$ However, while local approximations suffice to describe the nearly uniform average local magnetization in a ferromagnet, such approximations cannot be expected to fully reproduce the strong nonlocal correlations associated with spin waves in a noncollinear antiferromagnet. ${ }^{35}$

Faced with this difficulty, one might construct nonlocal, e.g., orbital-dependent functionals of the local variables $n(\mathbf{r})$ and $\mathbf{m}(\mathbf{r}) .{ }^{36} \mathrm{~A}$ simpler alternative is suggested by the procedure that, starting with spin-independent DFT, constructed SDFT and DFT for superconductors: a density sensitive to the ground-state correlations characteristic of the phenomenon under study is added to the list of fundamental variables. To the set $\left\{n_{\uparrow}(\mathbf{r}), n_{\downarrow}(\mathbf{r})\right\}$ of SDFT variables, noncollinear antiferromagnetism thus adds the staggered density $^{7,37,38}$

$$
\rho_{s}\left(\mathbf{r}, \mathbf{r}^{\prime}\right)=\left\langle\hat{\Psi}_{\uparrow}^{\dagger}(\mathbf{r}) \hat{\Psi}_{\downarrow}\left(\mathbf{r}^{\prime}\right)\right\rangle .
$$

The formal similarity between $\rho_{s}$ and the anomalous density in DFT for superconductors is not accidental: already in 1958 it was recognized that the restricted particle-hole transformation ${ }^{39,40}$

$$
\begin{aligned}
& \hat{\Psi}_{\uparrow}(\mathbf{r}) \mapsto \hat{\Psi}_{\uparrow}(\mathbf{r}), \\
& \hat{\Psi}_{\downarrow}(\mathbf{r}) \mapsto \hat{\Psi}_{\downarrow}^{\dagger}(\mathbf{r}),
\end{aligned}
$$

converts the BCS procedure into a mean-field theory of antiferromagnetism.

Central to the development of staggered DFT is a coupling between the staggered density and a staggered potential $S\left(\mathbf{r}, \mathbf{r}^{\prime}\right)$, a nonlocal generalization of the magnetic field $\mathbf{B}(\mathbf{r})$. In the resulting $\mathrm{KS}$ equations, the staggered density is coupled to the effective staggered potential

$$
S_{s}\left(\mathbf{r}, \mathbf{r}^{\prime}\right)=S_{\text {ext }}\left(\mathbf{r}, \mathbf{r}^{\prime}\right)+S_{H}\left(\mathbf{r}, \mathbf{r}^{\prime}\right)+S_{\mathrm{xc}}\left(\mathbf{r}, \mathbf{r}^{\prime}\right) .
$$

Exploring the analogy with the external pair potential in DFT for superconductivity, Refs. 7 and 37 conjectured that, more than a mathematical artifact, the first term on the right-hand side could be interpreted as a proximity effect - a potential induced near a noncollinear antiferromagnet. 
Two features distinguish staggered DFT from collinear SDFT. (i) The diagonal element $\left(\mathbf{r}^{\prime}=\mathbf{r}\right)$ of the staggered density determines the $x$ and $y$ components of the magnetization: $m_{x}(\mathbf{r})=\mu_{0}\left[\rho_{s}(\mathbf{r}, \mathbf{r})+\rho_{s}^{*}(\mathbf{r}, \mathbf{r})\right] \quad$ and $\quad m_{y}(\mathbf{r})=i \mu_{0}\left[\rho_{s}^{*}(\mathbf{r}, \mathbf{r})\right.$ $\left.-\rho_{s}(\mathbf{r}, \mathbf{r})\right]$. This restriction yields a formalism equivalent to local noncollinear SDFT. ${ }^{31-34}$ Even under this restriction, even for perfectly collinear states, noncollinear DFT is more powerful than standard (i.e., collinear) SDFT. In the latter, the specification of the quantization direction breaks rotational symmetry. The staggered density restores that symmetry: collinear SDFT is blind to, e.g., a magnetization along the $x$ axis since $n_{\uparrow}=n_{\downarrow}=0$. Staggered SDFT, by contrast, extracts the $x$ and $y$ components of the magnetization from $\rho_{s}$.

(ii) The nonlocal dependence $\rho_{s}=\rho_{s}\left(\mathbf{r}, \mathbf{r}^{\prime}\right)$ enhances the advantage of staggered DFT over SDFT and makes it more powerful than local noncollinear SDFT. To see this, it is sufficient to recall that the electron liquid possesses an instability against the formation of spin-density waves (SDW) ${ }^{41-43}$ The driving force of the Overhauser instability is the staggered Hartree interaction

$$
U_{x}\left[\rho_{s}\right]=-\int d^{3} r \int d^{3} r^{\prime} \frac{\left|\rho_{s}\left(\mathbf{r}, \mathbf{r}^{\prime}\right)\right|^{2}}{\left|\mathbf{r}-\mathbf{r}^{\prime}\right|} .
$$

This interaction, which formally appears as a Hartree term involving the staggered density, arises from evaluating the exchange diagram with two-component spinors. It tends to push the energy of the SDW state below that of the paramagnetic state. , $37^{3}$

Standard formulations of SDFT miss this energy reduction altogether; sophisticated approximations to the exchange-correlation functional are needed to account for it. ${ }^{36}$ By contrast, the right-hand side of Eq. (5) is trivially incorporated in the definition of the staggered DFT exchange-correlation energy functional; the epithet "staggered Hartree energy" emphasizes its formal similarity to the usual Hartree term while the subscript $x$ is a reminder of its physical origin in the exchange diagram. ${ }^{7,37,38}$ Its contribution $S_{H}\left(\mathbf{r}, \mathbf{r}^{\prime}\right)=\delta U_{x} / \delta \rho_{s}\left(\mathbf{r}, \mathbf{r}^{\prime}\right)$ to the effective Hartree potential $S_{s}\left(\mathbf{r}, \mathbf{r}^{\prime}\right)$ defines the second term on the right-hand side of Eq. (4). Staggered DFT has been shown to recover both the Overhauser instability in the exchange-only approximation and its suppression by correlation. ${ }^{7}$

\section{DENSITY-FUNCTIONAL FORMALISM FOR COMPETING ORDER PARAMETERS}

We now turn to a formulation that extends the results recapitulated in Sec. II to more complex ground states, with coexisting or competing order parameters. Our derivation following closely Ref. 18 for the superconducting order parameter and Ref. 7 for the noncollinear order parameter, we list but the key equations.

\section{A. Many-body Hamiltonian}

We consider an interacting Hamiltonian that includes external potentials coupled to all the physical observables of interest

$$
\begin{aligned}
\hat{H}= & \hat{T}+\hat{U}+\sum_{\sigma} \int d^{3} r \hat{n}_{\sigma}(\boldsymbol{r}) v_{\sigma}(\boldsymbol{r})+\int d^{3} x d^{3} y\left(\hat{\rho}_{S}(\boldsymbol{x}, \boldsymbol{y}) S(\boldsymbol{x}, \boldsymbol{y})\right. \\
& \left.-\hat{\chi}(\boldsymbol{x}, \boldsymbol{y}) \Delta^{*}(\boldsymbol{x}, \boldsymbol{y})-\sum_{m=-1}^{1} \hat{\chi}_{m}(\boldsymbol{x}, \boldsymbol{y}) \Delta_{m}^{*}(\boldsymbol{x}, \boldsymbol{y})+\text { H.c. }\right) .
\end{aligned}
$$

Here, the density operators $\hat{n}_{\sigma}(\boldsymbol{r}) \equiv \hat{\psi}_{\sigma}^{\dagger}(\boldsymbol{r}) \hat{\psi}_{\sigma}(\boldsymbol{r}), \quad \hat{\rho}_{s}\left(\boldsymbol{r}, \boldsymbol{r}^{\prime}\right)$ $\equiv \psi_{\uparrow}^{\dagger}(\boldsymbol{r}) \psi_{\downarrow}\left(\boldsymbol{r}^{\prime}\right), \hat{\chi}\left(\boldsymbol{r}, \boldsymbol{r}^{\prime}\right) \equiv\left[\psi(\boldsymbol{r}) \psi\left(\boldsymbol{r}^{\prime}\right)\right]_{s}$ (the singlet combination of the two field operators), and $\hat{\chi}_{m}\left(\boldsymbol{r}, \boldsymbol{r}^{\prime}\right) \equiv\left[\psi(\boldsymbol{r}) \psi\left(\boldsymbol{r}^{\prime}\right)\right]_{m}$ (the $m=-1,0$, and 1 triplet combinations of the field operators) are coupled to the external potentials $v_{\sigma}(\boldsymbol{r})=v(\boldsymbol{r})$ $+\sigma \mu_{B} B_{z}(\boldsymbol{r}), \quad S(\boldsymbol{x}, \boldsymbol{y}) \quad$ (the staggered potential $\left.{ }^{7}\right), \quad \Delta(\boldsymbol{x}, \boldsymbol{y})$ $=\Delta(\boldsymbol{y}, \boldsymbol{x})$ (the singlet pairing potential $\left.{ }^{18}\right)$, and $\Delta_{m}(\boldsymbol{x}, \boldsymbol{y})$ $=-\Delta_{m}(\boldsymbol{y}, \boldsymbol{x})$ (the $m$ component of the triplet pairing potential $\left.{ }^{18}\right)$, respectively. As usual, the kinetic-energy operator is $\hat{T} \equiv \sum_{\sigma=\uparrow \downarrow} \int d^{3} r \psi_{\sigma}^{\dagger}(\boldsymbol{r})\left(-\hbar^{2} \nabla^{2} / 2 m\right) \psi_{\sigma}(\boldsymbol{r})$, where $m$ is electronic mass and as in Refs. 6 and 18, we assume the following form for the electron-electron interaction:

$$
\begin{aligned}
\hat{U}:= & \frac{1}{2} \sum_{\beta_{1}, \alpha_{1}, \alpha_{2}, \beta_{2}} \int d^{3} y_{1} d^{3} x_{1} d^{3} x_{2} d^{3} y_{2} \hat{\psi}_{\beta_{1}}^{\dagger}\left(\boldsymbol{y}_{1}\right) \hat{\psi}_{\alpha_{1}}^{\dagger}\left(\boldsymbol{x}_{1}\right) \\
& \times U\left(\boldsymbol{y}_{1}, \beta_{1} ; \boldsymbol{x}_{1}, \alpha_{1} ; \boldsymbol{x}_{2}, \alpha_{2} ; \boldsymbol{y}_{2}, \beta_{2}\right) \hat{\psi}_{\alpha_{2}}\left(\boldsymbol{x}_{2}\right) \hat{\psi}_{\beta_{2}}\left(\boldsymbol{y}_{2}\right),
\end{aligned}
$$

where the electron-electron interaction comprises the Coulomb repulsion and a nonlocal spin-dependent term, needed to represent, e.g., the phonon-mediated attraction in conventional superconductors or a spin-fluctuation mediated interaction in unconventional ones

$$
\begin{aligned}
& U\left(\boldsymbol{y}_{1}, \beta_{1} ; \boldsymbol{x}_{1}, \alpha_{1} ; \boldsymbol{x}_{2}, \alpha_{2} ; \boldsymbol{y}_{2}, \beta_{2}\right) \\
& =\frac{q^{2}}{\left|\boldsymbol{x}_{1}-\boldsymbol{y}_{1}\right|} \delta\left(\boldsymbol{x}_{1}-\boldsymbol{x}_{2}\right) \delta\left(\boldsymbol{y}_{1}-\boldsymbol{y}_{2}\right) \delta_{\alpha_{1}, \alpha_{2}} \delta_{\beta_{1}, \beta_{2}} \\
& \quad+W\left(\boldsymbol{y}_{1}, \beta_{1} ; \boldsymbol{x}_{1}, \alpha_{1} ; \boldsymbol{x}_{2}, \alpha_{2} ; \boldsymbol{y}_{2}, \beta_{2}\right) .
\end{aligned}
$$

Since the following arguments make only implicit reference to $\hat{W}$, to be concise we have chosen a nonretarded potential to illustrate Eq. (8).

Lest the reader be puzzled by the asymmetry in our treatment of the pair density, which is resolved into a triplet and a singlet components, while the staggered density is not, we note that the restricted particle-hole transformation $\psi_{\uparrow}(\boldsymbol{r}) \mapsto \psi_{\uparrow}(\boldsymbol{r}), \psi_{\downarrow}(\boldsymbol{r}) \mapsto \psi_{\downarrow}^{\dagger}(\boldsymbol{r})$ would turn spins into isospins. Under this transformation, the Cooper pairs would comprise an isospin doublet coupled to an anomalous magnetic-field like potential, while the staggered density would decompose into an isospin singlet and an isospin triplet. Although the two approaches are mathematically equivalent, we find the language of spins more attractive for the present purposes than that of isospins.

\section{B. Kohn-Sham equations}

Given $\hat{U}$, the ground-state energy is a functional of the densities $n_{\sigma}(\sigma=\uparrow, \downarrow), \rho_{s}, \chi$, and $\chi_{m}(m=-1,0,1)$, which can be written as 


$$
\begin{aligned}
E\left[n_{\uparrow}, n_{\downarrow}, \rho_{s}, \chi, \chi_{m=0}, \chi_{m=+1}, \chi_{m=-1}\right] & =F_{H K}\left[n_{\uparrow}, n_{\downarrow}, \rho_{s}, \chi, \chi_{m=0}, \chi_{m=+1}, \chi_{m=-1}\right] \\
& +\sum_{\sigma} \int d^{3} r n_{\sigma}(\boldsymbol{r}) v_{\sigma}(\boldsymbol{r})+\int d^{3} x d^{3} y\left(\rho_{S}(\boldsymbol{x}, \boldsymbol{y}) S(\boldsymbol{x}, \boldsymbol{y})\right. \\
& \left.-\chi(\boldsymbol{x}, \boldsymbol{y}) \Delta^{*}(\boldsymbol{x}, \boldsymbol{y})-\sum_{m=-1}^{1} \chi_{m}(\boldsymbol{x}, \boldsymbol{y}) \Delta_{m}^{*}(\boldsymbol{x}, \boldsymbol{y})+\text { c.c. }\right),
\end{aligned}
$$

where the potential energy in the various external potentials has been written explicitly and the kinetic and interaction energies are combined into the Hohenberg-Kohn internalenergy functional $F_{\mathrm{HK}}$,

$$
F_{\mathrm{HK}}=\langle\hat{T}\rangle+\langle\hat{U}\rangle=T_{s}+U_{\mathrm{MF}}+E_{\mathrm{xc}} .
$$

In the last equation we defined the exchange-correlation functional $E_{\mathrm{xc}}=\langle\hat{T}\rangle-T_{s}+\langle\hat{U}\rangle-U_{\mathrm{MF}}$ in terms of the kinetic energy $T_{s}$ of a noninteracting system with densities $n_{\uparrow}, n_{\downarrow}, \rho_{s}, \chi, \chi_{m=0}, \chi_{m=+1}, \chi_{m=-1}$ and the mean-field approximation $U_{\mathrm{MF}}$ to the full interaction energy $\langle\hat{U}\rangle$. Formally, this mean-field approximation is given by

$$
\begin{aligned}
U_{\mathrm{MF}}= & \frac{1}{2} \sum_{\beta_{1}, \alpha_{1}, \alpha_{2}, \beta_{2}} \int d^{3} y_{1} d^{3} x_{1} d^{3} x_{2} d^{3} y_{2}\left\langle\hat{\psi}_{\beta_{1}}^{\dagger}\left(\boldsymbol{y}_{1}\right) \hat{\psi}_{\alpha_{1}}^{\dagger}\left(\boldsymbol{x}_{1}\right)\right\rangle U\left(\boldsymbol{y}_{1}, \beta_{1} ; \boldsymbol{x}_{1}, \alpha_{1} ; \boldsymbol{x}_{2}, \alpha_{2} ; \boldsymbol{y}_{2}, \beta_{2}\right)\left\langle\hat{\psi}_{\alpha_{2}}\left(\boldsymbol{x}_{2}\right) \hat{\psi}_{\beta_{2}}\left(\boldsymbol{y}_{2}\right)\right\rangle \\
& +\frac{1}{2} \sum_{\beta_{1}, \alpha_{1}, \alpha_{2}, \beta_{2}} \int d^{3} y_{1} d^{3} x_{1} d^{3} x_{2} d^{3} y_{2}\left\langle\hat{\psi}_{\beta_{1}}^{\dagger}\left(\boldsymbol{y}_{1}\right) \hat{\psi}_{\beta_{2}}\left(\boldsymbol{y}_{1}\right)\right\rangle U\left(\boldsymbol{y}_{1}, \beta_{1} ; \boldsymbol{x}_{1}, \alpha_{1} ; \boldsymbol{x}_{2}, \alpha_{2} ; \boldsymbol{y}_{2}, \beta_{2}\right)\left\langle\hat{\psi}_{\alpha_{1}}^{\dagger}\left(\boldsymbol{x}_{1}\right) \hat{\psi}_{\alpha_{2}}\left(\boldsymbol{x}_{2}\right)\right\rangle \\
& -\frac{1}{2} \sum_{\beta_{1}, \alpha_{1}, \alpha_{2}, \beta_{2}} \int d^{3} y_{1} d^{3} x_{1} d^{3} x_{2} d^{3} y_{2}\left\langle\hat{\psi}_{\beta_{1}}^{\dagger}\left(\boldsymbol{y}_{1}\right) \hat{\psi}_{\alpha_{2}}\left(\boldsymbol{x}_{2}\right)\right\rangle U\left(\boldsymbol{y}_{1}, \beta_{1} ; \boldsymbol{x}_{1}, \alpha_{1} ; \boldsymbol{x}_{2}, \alpha_{2} ; \boldsymbol{y}_{2}, \beta_{2}\right)\left\langle\hat{\psi}_{\alpha_{1}}^{\dagger}\left(\boldsymbol{x}_{1}\right) \hat{\psi}_{\beta_{2}}\left(\boldsymbol{y}_{2}\right)\right\rangle .
\end{aligned}
$$

Some contributions to $U_{\mathrm{MF}}$ have appeared before in more restricted formulations, such as the conventional Hartree term

$$
E_{H}=\frac{1}{2} \int d^{3} x \int d^{3} y \frac{n(\mathbf{x}) n(\mathbf{y})}{|\mathbf{x}-\mathbf{y}|}
$$

the anomalous Hartree term

$$
E_{H}^{a}=\int d^{3} x \int d^{3} y \frac{|\chi(\mathbf{x}, \mathbf{y})|^{2}}{|\mathbf{x}-\mathbf{y}|},
$$

and the staggered Hartree term

$$
E_{H}^{s}=-\int d^{3} x \int d^{3} y \frac{\left|\rho_{s}(\mathbf{x}, \mathbf{y})\right|^{2}}{|\mathbf{x}-\mathbf{y}|} \equiv U_{x}\left[\rho_{s}\right] .
$$

We stress that while $E_{H}^{a}$ is repulsive and thus detrimental to superconductivity, ${ }^{24} U_{x}\left[\rho_{s}\right]$ is attractive and favors formation of spin-density waves. ${ }^{7,37,38}$

The KS procedure, applied to this total-energy functional, leads to single-particle equations for the four-component spinor

$$
\Phi^{\ell}(\boldsymbol{r})=\left[u_{\uparrow}^{\ell}(\boldsymbol{r}), u_{\downarrow}^{\ell}(\boldsymbol{r}), v_{\uparrow}^{\ell}(\boldsymbol{r}), v_{\downarrow}^{\ell}(\boldsymbol{r})\right]^{T},
$$

with particle (hole) wave functions $u_{\sigma}(\boldsymbol{r})\left[v_{\sigma}(\boldsymbol{r})\right]$ for each electron spin, $\sigma=\uparrow, \downarrow$. The symbol $T$ indicates transposition, while the superscript $\ell$ labels the self-consistent solutions of the KS equations, the first of which is

$$
H^{(s)}\left[w^{(s)}\right] \Phi^{\ell}=\varepsilon_{\ell} \Phi^{\ell},
$$

where $w^{(s)}$ denotes the set of the seven potentials $\left\{v_{\sigma}^{(s)}, S^{(s)}, \Delta^{(s)}, \Delta_{m}^{(s)}\right\} \quad(\sigma=\uparrow, \downarrow, m=-1,0,1)$. The KS Hamiltonian is

$$
H^{(s)}=\left(\begin{array}{cccc}
h+v_{\uparrow, s} & \hat{S}^{(s)} & -2 \hat{\Delta}_{1}^{(s)} & -\hat{\Delta}^{(s)}-\hat{\Delta}_{0}^{(s)} \\
\hat{S}^{(s) \dagger} & h+v_{\downarrow, s} & \hat{\Delta}^{(s)}-\hat{\Delta}_{0}^{(s)} & -2 \hat{\Delta}_{-1}^{(s)} \\
2 \hat{\Delta}_{1}^{(s) *} & \hat{\Delta}_{0}^{(s) *}+\hat{\Delta}^{(s) *} & -h-v_{\uparrow, s} & -\hat{S}^{(s) *} \\
\hat{\Delta}_{0}^{(s) *}-\hat{\Delta}^{(s) *} & 2 \hat{\Delta}_{-1}^{(s) *} & -\hat{S}^{(s) T} & -h-v_{\downarrow, s}
\end{array}\right),
$$

with the shorthands $h$ for $-\hbar^{2} \nabla^{2} / 2 m$ and $\hat{S}^{(s)}, \hat{\Delta}^{(s)}$, and $\hat{\Delta}_{m}^{(s)}$ for the integral operators associated with the nonlocal potentials $S^{(s)}\left(\boldsymbol{r}, \boldsymbol{r}^{\prime}\right), \Delta^{(s)}\left(\boldsymbol{r}, \boldsymbol{r}^{\prime}\right)$, and $\Delta_{m}^{(s)}\left(\boldsymbol{r}, \boldsymbol{r}^{\prime}\right)$ by

$$
\begin{aligned}
& \hat{S}^{(s)}: f(\boldsymbol{x}) \mapsto \int d^{3} y S^{(s)}(\boldsymbol{x}, \boldsymbol{y}) f(\boldsymbol{y}), \\
& \hat{\Delta}^{(s)}: f(\boldsymbol{x}) \mapsto \int d^{3} y \Delta^{(s)}(\boldsymbol{x}, \boldsymbol{y}) f(\boldsymbol{y}), \\
& \hat{\Delta}_{m}^{(s)}: f(\boldsymbol{x}) \mapsto \int d^{3} y \Delta_{m}^{(s)}(\boldsymbol{x}, \boldsymbol{y}) f(\boldsymbol{y}) .
\end{aligned}
$$

The effective single-body potentials are defined by

$$
v_{\sigma s}=v_{\sigma}+\frac{\delta U_{\mathrm{MF}}}{\delta n_{\sigma}}+\frac{\delta E_{\mathrm{xc}}}{\delta n_{\sigma}},
$$




$$
\begin{gathered}
S^{(s)}=S+\frac{\delta U_{\mathrm{MF}}}{\delta \rho_{s}}+\frac{\delta E_{\mathrm{xc}}}{\delta \rho_{s}}, \\
\Delta^{(s)}=\Delta+\frac{\delta U_{\mathrm{MF}}}{\delta \chi}+\frac{\delta E_{\mathrm{xc}}}{\delta \chi}, \\
\Delta_{m}^{(s)}=\Delta_{m}+\frac{\delta U_{\mathrm{MF}}}{\delta \chi_{m}}+\frac{\delta E_{\mathrm{xc}}}{\delta \chi_{m}} .
\end{gathered}
$$

The derivation of Eq. (17) from Eq. (9) is analogous to that of the simpler KS Hamiltonians in Refs. 6 and 7. First, we note that minimization of the interacting Hamiltonian of Eq. (6) with respect to $n_{\sigma}$ results in the Euler equation

$$
\frac{\delta T_{s}}{\delta n_{\sigma}(\mathbf{r})}+\frac{\delta U_{\mathrm{MF}}}{\delta n_{\sigma}(\mathbf{r})}+\frac{\delta E_{\mathrm{xc}}}{\delta n_{\sigma}(\mathbf{r})}+v_{\sigma}(\mathbf{r})=0
$$

and similar equations for the minimization with respect to the other densities.

In a second step, we construct a noninteracting manybody system with Hamiltonian $\hat{H}_{s}$ obtained by subtracting $\hat{U}$ from the interacting Hamiltonian of Eq. (6) and replacing the external potentials $v_{\sigma}, S, \Delta$, and $\Delta_{m}$ with $v_{\sigma, s}, S^{(s)}, \Delta^{(s)}$, and $\Delta_{m}^{(s)}$, respectively. These self-consistent potentials are chosen such that the noninteracting system has the same densities $n_{\uparrow}$, $n_{\downarrow}, \rho_{s}, \chi$, and $\chi_{m}$ as given by the original Hamiltonian $\hat{H}$. Minimization of $\hat{H}_{s}$ with respect to $n_{\sigma}$ leads to the Euler equation

$$
\frac{\delta T_{s}}{\delta n_{\sigma}(\mathbf{r})}+v_{\sigma, s}(\mathbf{r})=0 .
$$

The two Euler equations can be combined to yield Eq. (19a). In the same manner, minimization with respect to the other densities yields Eqs. (19b)-(19d). To solve the effective problem, we need to diagonalize $\hat{H}_{s}$. We achieve this by a linear (Bogolubov) transformation from the electron creation and annihilation operators, $\hat{\psi}_{\sigma}^{\dagger}(\mathbf{r})$ and $\hat{\psi}_{\sigma}(\mathbf{r})$, to new fermionic creation and annihilation operators $\hat{\gamma}_{l}^{\dagger}, \hat{\gamma}_{l}$,

$$
\hat{\psi}_{\sigma}(\mathbf{r})=\sum_{l}\left[u_{\sigma}^{l}(\mathbf{r}) \hat{\gamma}_{l}+v_{\sigma}^{l}(\mathbf{r})^{*} \hat{\gamma}_{l}^{\dagger}\right] \text {. }
$$

This transformation renders the effective single-particle Hamiltonian in diagonal form $\hat{H}_{s}=$ const $+\Sigma_{l} \varepsilon_{l} \hat{\gamma}_{l}^{\dagger} \hat{\gamma}_{l}$. The single-particle energies $\varepsilon_{l}$ are the eigenvalues of the KS Hamiltonian (17), while the coefficients $u_{\sigma}^{l}$ and $v_{\sigma}^{l}$ of the Bogolubov transformation are the components of the spinors $\Phi^{l}$.

In terms of these coefficients, the ground-state densities are given by

$$
\begin{gathered}
n_{\sigma}(\boldsymbol{r})=\sum_{\ell: \varepsilon_{\ell}<0}\left|u_{\sigma}^{\ell}(\boldsymbol{r})\right|^{2}+\sum_{\ell: \varepsilon_{\ell}>0}\left|v_{\sigma}^{\ell}(\boldsymbol{r})\right|^{2} \quad(\sigma=\uparrow, \downarrow), \\
\rho_{s}(\boldsymbol{x}, \boldsymbol{y})=\sum_{\ell: \varepsilon_{\ell}<0} u_{\uparrow}^{\ell *}(\boldsymbol{x}) u_{\downarrow}^{\ell}(\boldsymbol{y})+\sum_{\ell: \varepsilon_{\ell}>0} v_{\uparrow}^{\ell}(\boldsymbol{x}) v_{\downarrow}^{\ell *}(\boldsymbol{y}),
\end{gathered}
$$

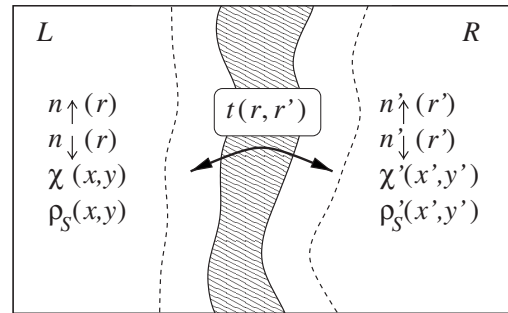

FIG. 1. The physical system under consideration (see text): the shaded area represents the physically inaccessible region across which tunneling can take place, while the dashed lines indicate the extent of the $L$ and $R$ regions on which the tunneling amplitude $t\left(\boldsymbol{r}, \boldsymbol{r}^{\prime}\right)$ is important.

$$
\begin{aligned}
\chi(\boldsymbol{x}, \boldsymbol{y}) & =\sum_{\ell: \varepsilon_{\ell}<0}\left[v^{\ell *}(\boldsymbol{x}) u^{\ell}(\boldsymbol{y})\right]_{s}+\sum_{\ell: \varepsilon_{\ell}>0}\left[u^{\ell}(\boldsymbol{x}) v^{\ell *}(\boldsymbol{y})\right]_{s}, \\
\chi_{m}(\boldsymbol{x}, \boldsymbol{y}) & =\sum_{\ell: \varepsilon_{\ell}<0}\left[v^{\ell *}(\boldsymbol{x}) u^{\ell}(\boldsymbol{y})\right]_{m}+\sum_{\ell: \varepsilon_{\ell}>0}\left[u^{\ell}(\boldsymbol{x}) v^{\ell *}(\boldsymbol{y})\right]_{m} .
\end{aligned}
$$

The ground-state energy is then obtained by computing the functional $E\left[n_{\uparrow}, n_{\downarrow}, \rho_{s}, \chi, \chi_{m=0}, \chi_{m=+1}, \chi_{m=-1}\right]$.

The KS Eqs. (16), (17), (18a)-(18c), and (19a)-(19d) determine the ground-state energy and densities for external potentials ranging from microscopically to macroscopically inhomogeneous, as well as from periodic to disordered. Our interest in the proximity effect focuses the following discussion on the inhomogeneities associated with a onedimensional discontinuity separating two semi-infinite regions.

\section{SPECIALIZATION TO INTERFACES}

We consider two semi-infinite spatial regions, labeled $L$ (left) and $R$ (right), separated by a potential barrier that is wide in comparison with the range of the interaction $\hat{U}$ in either $L$ or $R$. Though high, the barrier is finite and hence allows electronic tunneling between $L$ and $R$. As Fig. 1 indicates, we denote the position in $L(R)$ by $\boldsymbol{r}\left(\boldsymbol{r}^{\prime}\right)$. To save space, we will indicate the densities and potentials on the $R$ semispace by a prime. Thus, e.g., $v(\boldsymbol{r} \in L)[v(\boldsymbol{r} \in R)]$ will be denoted $v\left(v^{\prime}\right)$.

Our model Hamiltonian reads

$$
\hat{H}=\hat{H}_{L}+\hat{H}_{T}+\hat{H}_{R}
$$

Here, $\hat{H}_{L}\left(\hat{H}_{R}\right)$ are of the form $\hat{H}+\hat{V}$, where $\hat{H}$ is defined in Eq. (6) and $\hat{V}$ is an infinite barrier centered at the interface between $L(R)$ and the tunneling region, which enforces orthogonality between the eigenfunctions of $\hat{H}_{L}$ and $\hat{H}_{R} \cdot{ }^{44}$ The phenomenological tunneling Hamiltonian

$$
\hat{H}_{T}=\sum_{\sigma} \int_{L} d^{3} r \int_{R} d^{3} r^{\prime}\left[\hat{\psi}_{\sigma}^{\dagger}(\boldsymbol{r}) t\left(\boldsymbol{r}, \boldsymbol{r}^{\prime}\right) \hat{\psi}_{\sigma}\left(\boldsymbol{r}^{\prime}\right)+\text { H.c. }\right]
$$


We complete the phenomenological description of the interface by assuming that the only effect of the tunneling term $\hat{H}_{T}$ in the Hamiltonian (24) is to make the KS equations become

$$
\left(\begin{array}{cc}
H^{(s)}\left[w^{(s)}\right] & H_{T}^{(s)} \\
H_{T}^{(s) \dagger} & H^{(s)}\left[w^{\left.(s)^{\prime}\right]}\right.
\end{array}\right)\left(\begin{array}{l}
\Phi_{L}^{\nu} \\
\Phi_{R}^{\nu}
\end{array}\right)=\varepsilon_{\nu}\left(\begin{array}{c}
\Phi_{L}^{\nu} \\
\Phi_{R}^{\nu}
\end{array}\right),
$$

where the tunneling KS Hamiltonian $H_{T}^{(s)}$ is defined by

$$
H_{T}^{(s)}=\left(\begin{array}{cccc}
\hat{t} & 0 & 0 & 0 \\
0 & \hat{t} & 0 & 0 \\
0 & 0 & -\hat{t}^{*} & 0 \\
0 & 0 & 0 & -\hat{t}^{*}
\end{array}\right) .
$$

The integral operator $\hat{t}$ is given by $\hat{t}: f(\boldsymbol{r}) \mapsto \int d^{3} r^{\prime} t\left(\boldsymbol{r}, \boldsymbol{r}^{\prime}\right) f\left(\boldsymbol{r}^{\prime}\right)$.

Our phenomenological description of the barrier contrasts with the microscopic treatment of the semi-infinite regions. Instead of deriving and solving Kohn-Sham equations for the shaded region in Fig. 1, we prefer to follow Prange's prescription ${ }^{44}$ even if we have to rely on phenomenological considerations to define the tunneling matrix $t\left(\mathbf{r}, \mathbf{r}^{\prime}\right)$. This simplification focuses our analysis on the interaction between the $L$ and $R$ regions. Under appropriate circumstances, as shown below, it leads to an independent set of Kohn-Sham equations for each region - a result combining mathematical convenience with physical appeal. Under such circumstances, to determine the densities in one of the regions, we can rely on first-principles calculations in which the external potential captures phenomenologically the influence of the material across the barrier.

\section{A. Decoupled Hamiltonians}

The KS Eq. (26) describes the electronic states of $L$ and $R$. Let us now derive a set of $\mathrm{KS}$ equations that describe, formally, only the states of $R$, but take into account the influence on such states of the charge, spin, and superconducting order that are potentially present in $L$.

For $\boldsymbol{r} \in L$, since the eigenfunctions of the KS Hamiltonian with $\hat{H}_{T}=0$ form a complete set, we can write

$$
\Phi_{L}^{\nu}=\sum_{\ell}\left(\widetilde{\Phi}_{L}^{\ell \dagger}, \Phi_{L}^{\nu}\right) \tilde{\Phi}_{L}^{\ell},
$$

where the tildes denote the absence of tunneling and the scalar product $\left(\widetilde{\Phi}_{L}^{\ell \dagger}, \Phi_{L}^{\nu}\right)$ comprises integration over the spatial variable and summation over spinor indices.
Insertion into the second row of Eq. (26) then yields

$$
\sum_{\ell}\left(\widetilde{\Phi}_{L}^{\ell \dagger}, \Phi_{L}^{\nu}\right) H_{T}^{(s)} \widetilde{\Phi}_{L}^{\ell}+H^{(s)}\left[w^{(s)^{\prime}}\right] \Phi_{R}^{\nu}=\varepsilon_{\nu} \Phi_{R}^{\nu}
$$

To eliminate $\Phi_{L}^{v}$ from this equation, we first write the eigenvalue equation defining $\widetilde{\Phi}_{L}^{\ell}$, i.e., the first row of Eq. (26) with $H_{T}^{(s)}=0$,

$$
H^{(s)}\left[w^{(s)}\right] \widetilde{\Phi}_{L}^{\ell}=\widetilde{\varepsilon}_{L}^{\ell} \widetilde{\Phi}_{L}^{\ell},
$$

and multiply it on the left by $\Phi_{L}^{\nu \dagger}$. Hermitian conjugation then yields

$$
\widetilde{\Phi}_{L}^{\ell \dagger} H^{(s)}\left[w^{(s)}\right] \Phi_{L}^{\nu}=\widetilde{\varepsilon}_{L}^{\ell} \widetilde{\Phi}_{L}^{\ell \dagger} \Phi_{L}^{\nu} .
$$

Next, we multiply the first row of Eq. (26) by $\widetilde{\Phi}_{L}^{\ell \dagger}$ on the left

$$
\widetilde{\Phi}_{L}^{\ell \dagger} H_{T}^{(s)} \Phi_{R}^{\nu}+\widetilde{\Phi}_{L}^{\ell \dagger} H^{(s)}\left[w^{(s)}\right] \Phi_{L}^{\nu}=\varepsilon_{\nu} \widetilde{\Phi}_{L}^{\ell \dagger} \Phi_{L}^{\nu}
$$

We then subtract Eq. (31) from this result, solve for $\widetilde{\Phi}_{L}^{\ell \dagger} \Phi_{L}^{\nu}$, and integrate over the spatial variable to find that

$$
\left(\widetilde{\Phi}_{L}^{\ell \dagger}, \Phi_{L}^{\nu}\right)=\frac{1}{\varepsilon_{\nu}-\widetilde{\varepsilon}_{L}^{\ell}}\left(\widetilde{\Phi}_{L}^{\ell \dagger}, H_{T}^{(s)} \Phi_{R}^{\nu}\right) .
$$

We can now substitute the right-hand side for the scalar product on the left-hand side of Eq. (29) and exploit the completeness of the wave functions $\widetilde{\varphi}_{L}^{\ell}(l=1,2, \ldots)$ to define the effective external Hamiltonian

$$
H_{\mathrm{ext}}\left(\varepsilon_{\nu}\right)=\sum_{\ell} H_{T}^{(s)} \widetilde{\Phi}_{L}^{\ell} \frac{1}{\varepsilon_{\nu}-\widetilde{\varepsilon}_{L}^{\ell}} \widetilde{\Phi}_{L}^{\ell \dagger} H_{T}^{(s)},
$$

so that Eq. (29) defines a KS eigenvalue equation for $\boldsymbol{r}^{\prime}$ $\in R$,

$$
\left(H^{(s)}\left[w^{(s)^{\prime}}\right]+H_{\mathrm{ext}}\left(\varepsilon_{\nu}\right)\right) \Phi_{R}^{\nu}=\varepsilon_{\nu} \Phi_{R}^{\nu} .
$$

More explicitly, given that, if $\left(\widetilde{u}_{\uparrow}, \widetilde{u}_{\downarrow}, \widetilde{v}_{\uparrow}, \widetilde{v}_{\downarrow}\right)^{T}$ is an eigenvector, with eigenvalue $\widetilde{\varepsilon}$, then $\left(\widetilde{v}_{\uparrow}, \widetilde{v}_{\downarrow}, \widetilde{u}_{\uparrow}, \widetilde{u}_{\downarrow}\right)^{\dagger}$ is also an eigenvector, with eigenvalue $-\widetilde{\varepsilon}$, we can cast the effective external potential in the form

$$
H_{\mathrm{ext}}\left(\varepsilon_{\nu}\right)=\left(\begin{array}{cccc}
\hat{v}_{\uparrow}^{(s)}\left(\varepsilon_{\nu}\right) & \hat{S}^{(s)}\left(\varepsilon_{\nu}\right) & -2 \hat{\Delta}_{1}^{(s)}\left(\varepsilon_{\nu}\right) & -\left(\hat{\Delta}^{(s)}+\hat{\Delta}_{0}^{(s)}\right)\left(\varepsilon_{\nu}\right) \\
\hat{S}^{(s) \dagger}\left(\varepsilon_{\nu}\right) & \hat{v}_{\downarrow}^{(s)}\left(\varepsilon_{\nu}\right) & \left(\hat{\Delta}^{(s)}-\hat{\Delta}_{0}^{(s)}\right)\left(\varepsilon_{\nu}\right) & -2 \hat{\Delta}_{-1}^{(s)}\left(\varepsilon_{\nu}\right) \\
2 \hat{\Delta}_{1}^{(s) *}\left(-\varepsilon_{\nu}\right) & \left(\hat{\Delta}_{0}^{(s) *}+\hat{\Delta}^{(s) *}\right)\left(-\varepsilon_{\nu}\right) & -\hat{v}_{\uparrow}^{(s)}\left(-\varepsilon_{\nu}\right) & -\hat{S}^{(s) *}\left(-\varepsilon_{\nu}\right) \\
\left(\hat{\Delta}_{0}^{(s) *}-\hat{\Delta}^{(s) *}\right)\left(-\varepsilon_{\nu}\right) & 2 \hat{\Delta}_{-1}^{(s) *}\left(-\varepsilon_{\nu}\right) & -\hat{S}^{(s) T}\left(-\varepsilon_{\nu}\right) & -\hat{v}_{\downarrow}^{(s)}\left(-\varepsilon_{\nu}\right)
\end{array}\right),
$$




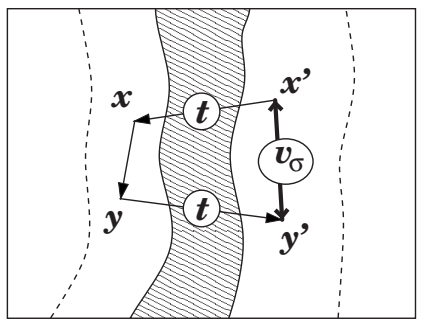

FIG. 2. The external nonlocal "potential" of Eq. (37a) induced in the $R$ region describes new coupling between points $\boldsymbol{x}^{\prime}$ and $\boldsymbol{y}^{\prime}$ that takes into account processes in which an electron would tunnel across the barrier, propagate inside the differently ordered electron liquid in the $L$ region, and then tunnel back into $R$ (see text).

where the integral operators $\hat{v}_{\sigma}\left(\varepsilon_{v}\right), \hat{S}\left(\varepsilon_{\nu}\right), \hat{\Delta}\left(\varepsilon_{\nu}\right)$, and $\hat{\Delta}_{m}\left(\varepsilon_{\nu}\right)$ $(m=-1,0,1)$ have the following kernels:

$$
v_{\sigma}\left(\varepsilon_{\nu} ; \boldsymbol{x}^{\prime}, \boldsymbol{y}^{\prime}\right)=\int_{L} d^{3} x t\left(\boldsymbol{x}^{\prime}, \boldsymbol{x}\right) \sum_{\ell} \int_{L} d^{3} y \frac{\widetilde{u}_{L, \sigma}^{\ell}(\boldsymbol{x}) \widetilde{u}_{L, \sigma}^{\ell *}(\boldsymbol{y})}{\varepsilon_{\nu}-\widetilde{\varepsilon}_{L, l}} t\left(\boldsymbol{y}, \boldsymbol{y}^{\prime}\right),
$$

$$
S\left(\varepsilon_{\nu} ; \boldsymbol{x}^{\prime}, \boldsymbol{y}^{\prime}\right)=\int_{L} d^{3} x t\left(\boldsymbol{x}^{\prime}, \boldsymbol{x}\right) \sum_{\ell} \int_{L} d^{3} y \frac{\widetilde{u}_{L, \uparrow}^{\ell}(\boldsymbol{x}) \widetilde{u}_{L, \downarrow}^{\ell *}(\boldsymbol{y})}{\varepsilon_{\nu}-\widetilde{\varepsilon}_{L, l}} t\left(\boldsymbol{y}, \boldsymbol{y}^{\prime}\right),
$$

$$
\begin{aligned}
\Delta\left(\varepsilon_{\nu} ; \boldsymbol{x}^{\prime}, \boldsymbol{y}^{\prime}\right)= & \int_{L} d^{3} x t\left(\boldsymbol{x}^{\prime}, \boldsymbol{x}\right) \sum_{\ell} \int_{L} d^{3} y \frac{\left[\widetilde{u}_{L}^{\ell}(\boldsymbol{x}) \widetilde{v}_{L}^{\ell *}(\boldsymbol{y})\right]_{s}}{\varepsilon_{\nu}-\widetilde{\varepsilon}_{L, l}} \\
& \times(-1) t^{*}\left(\boldsymbol{y}, \boldsymbol{y}^{\prime}\right), \\
\Delta_{m}\left(\varepsilon_{\nu} ; \boldsymbol{x}^{\prime}, \boldsymbol{y}^{\prime}\right)= & \int_{L} d^{3} x t\left(\boldsymbol{x}^{\prime}, \boldsymbol{x}\right) \sum_{\ell} \int_{L} d^{3} y \frac{\left[\widetilde{u}_{L}^{\ell}(\boldsymbol{x}) \widetilde{v}_{L}^{\ell *}(\boldsymbol{y})\right]_{m}}{\varepsilon_{\nu}-\widetilde{\varepsilon}_{L, l}} \\
& \times(-1) t^{*}\left(\boldsymbol{y}, \boldsymbol{y}^{\prime}\right) .
\end{aligned}
$$

The effective external potential $H_{\text {ext }}\left(\varepsilon_{\nu}\right)$ expresses mathematically the proximity effect. The nonlocal effective potential $v_{\sigma}\left(\varepsilon_{\nu} ; \boldsymbol{x}^{\prime}, \boldsymbol{y}^{\prime}\right)$ in Eq. (37a) describes the virtual transition from point $\boldsymbol{x}^{\prime}$ to $\boldsymbol{y}^{\prime}$ depicted in Fig. 2, with intermediate tunneling to point $\boldsymbol{x}$ and propagation to point $\boldsymbol{y}$. The righthand sides of Eqs. (37b)-(37d) have analogous interpretations, the propagation in $L$ now involving a spin flip [Eq. (37b)] or spin-conserving scattering into a hole state [Eqs. (37c) and (37d)], respectively.

\section{B. General aspects of the decoupled Hamiltonian}

Equations (37a)-(37d) decompose a pair of quantum liquids, differently ordered and coupled by tunneling, into two uncoupled liquids subject to effective, energy-dependent external fields. The right-hand side of each equality depends only on $\widetilde{\Phi}_{L}^{\ell}$, not on $\Phi_{R}^{\nu}$ or on $\Phi_{L}^{\nu}$. In words, the effective fields induced by the proximity effect on one half-space depend only on the solutions of the KS equations on the opposite side, unperturbed by tunneling.
In general, the effective fields also depend on the energies $\varepsilon_{v}$. Whether it is easier to diagonalize energy-independent coupled Hamiltonians or energy-dependent uncoupled ones then depends on specific aspects of the problem under study. If a low barrier separates degenerate states, the coupledHamiltonian formalism is natural and mathematically convenient. As pointed out in Ref. 6, however, if the energy spectrum $\tilde{\boldsymbol{\epsilon}}$ on one of the sides is bounded by a gap while that on the other side is not, then the low KS eigenvalues $\varepsilon_{\nu}$ can be neglected in the denominators of the sums on the right-hand sides of Eqs. (37a)-(37d) and the potentials become energy independent. In this case, the treatment of the decoupled Hamiltonians is clearly more efficient than the solution of the coupled KS equations. From a conceptual viewpoint, moreover, the emergence of proximity-induced effective potentials that give rise to superconductivity or antiferromagnetism is appealing.

In the analysis of infinite systems, at the formal level, DFT starts out by introducing a fictitious external potential, set equal to zero after the KS equations are derived. Equation (6) is an example. To solve the KS equations, at the operational level, one likewise seeds the self-consistent loop with an artificial external potential. In the vicinity of an ordered system, as Eqs. (37a)-(37d) show, this mathematical expedient is neither formally nor operationally necessary. One can therefore interpret the spontaneous ordering of an infinite system as a residual consequence of a proximity effect. We see that this interpretation, first offered in connection with superconductors in Ref. 6, describes equally well magnetic, superconducting, and coexisting states.

In spite of the complex form on the right-hand side of Eq. (36), our attention to spin in the definition of the Cooper-pair densities guarantees that the KS equations couple only the singlet (triplet) density to the singlet (triplet) potential. As the analyses in Secs. IV C and IV D will show, if a conventional superconductor is coupled to a noncollinear antiferromagnet by an interface through which electrons can tunnel, the resulting proximity potential will induce the formation of singlet Cooper pairs in the antiferromagnetic material. Likewise, on the superconducting side, the induced staggered field scatters the Cooper pairs into other singlet states.

\section{Special case: Superconductivity}

This subsection and the next examine Eqs. (37a)-(37d) in two particularly simple situations. Here, we detail the first arrangement, which couples an unspecified material $(R)$ to a singlet superconductor $(L)$. We take the latter to be nonmagnetic, so that $v_{s, \uparrow}-v_{s, \downarrow}=S_{s}=0$.

To determine the unperturbed KS eigenstates in $L$, which contribute to the right-hand sides of Eqs. (37a)-(37d), we set $H_{T}^{s}=0$. A Bogolubov-Valatin transformation then diagonalizes the matrix on the right-hand side of the resulting Eq. (17). The eigenspinors have the form

$$
\begin{gathered}
\widetilde{\Phi}_{L}^{\alpha \ell}(\mathbf{r}) \equiv\left(\begin{array}{c}
\widetilde{u}_{L, \uparrow}^{\alpha \ell}(\mathbf{r}) \\
\widetilde{u}_{L, \downarrow}^{\alpha \ell}(\mathbf{r}) \\
\widetilde{v}_{L, \uparrow}^{\alpha \ell}(\mathbf{r}) \\
\tilde{v}_{L, \downarrow}^{\alpha \ell}(\mathbf{r})
\end{array}\right)=\left(\begin{array}{c}
u_{\ell}(\mathbf{r}) \\
0 \\
0 \\
v_{\ell}(\mathbf{r})
\end{array}\right) \delta_{\alpha, 1}+\left(\begin{array}{c}
0 \\
u_{\ell}(\mathbf{r}) \\
-v_{\ell}(\mathbf{r}) \\
0
\end{array}\right) \delta_{\alpha, 2} \\
(\alpha=1,2) .
\end{gathered}
$$


The amplitudes $u_{\ell}(\mathbf{r})$ and $v_{\ell}(\mathbf{r})$ are solutions of the Bogolubov-de Gennes equations [i.e., Eq. 7 in Ref. 6]. The invariance of the $L$ Hamiltonian under the inversion $z \mapsto-z$ makes the KS eigenvalues independent of the spin index $\alpha$, i.e., $\widetilde{\varepsilon}_{L}^{\alpha \ell}=\widetilde{\varepsilon}_{\ell}$. The eigenvalues moreover change sign under the transformation $\left(\widetilde{u}_{\uparrow}, \widetilde{u}_{\downarrow}, \widetilde{v}_{\uparrow}, \widetilde{v}_{\downarrow}\right)^{T_{1}} \mapsto\left(\widetilde{v}_{\uparrow}, \widetilde{v}_{\downarrow}, \widetilde{u}_{\uparrow}, \widetilde{u}_{\downarrow}\right)^{\dagger}$. These two symmetries considered, and the eigenfunctions on the right-hand side of Eq. (38) substituted on the right-hand sides of Eqs. (37a)-(37d), we find that

$$
v_{\sigma}\left(\varepsilon_{\nu} ; \boldsymbol{x}^{\prime}, \boldsymbol{y}^{\prime}\right)=\int_{L} d^{3} x t\left(\boldsymbol{x}^{\prime}, \boldsymbol{x}\right) \sum_{\ell} \int_{L} d^{3} y \frac{u_{\ell \sigma}(\boldsymbol{x}) u_{\ell \sigma}^{*}(\boldsymbol{y})}{\varepsilon_{\nu}-\widetilde{\varepsilon}_{\ell}} t\left(\boldsymbol{y}, \boldsymbol{y}^{\prime}\right),
$$

$$
\Delta\left(\varepsilon_{\nu} ; \boldsymbol{x}^{\prime}, \boldsymbol{y}^{\prime}\right)=\int_{L} d^{3} x t\left(\boldsymbol{x}^{\prime}, \boldsymbol{x}\right) \sum_{\ell} \int_{L} d^{3} y\left[\frac{u_{\ell}(\boldsymbol{x}) v_{\ell}^{*}(\boldsymbol{y})}{\widetilde{\varepsilon}_{\ell}-\varepsilon_{\nu}}\right]_{s} t^{*}\left(\boldsymbol{y}, \boldsymbol{y}^{\prime}\right),
$$

$$
S\left(\varepsilon_{\nu} ; \boldsymbol{x}^{\prime}, \boldsymbol{y}^{\prime}\right)=\Delta_{m}\left(\varepsilon_{\nu} ; \boldsymbol{x}^{\prime}, \boldsymbol{y}^{\prime}\right)=0 \quad(m=-1,0,1) .
$$

Thus the superconductivity in $L$ induces only a normal and a singlet pairing potential in $R$. When $R$ is a semiinfinite, normal metal, we recover the superconductor-DFT result. ${ }^{6}$ Equation (39a) is the nonlocal normal potential alluded to, but not given explicitly, in footnote 10 of Ref. 6 .

\section{Special case: Magnetism}

As a second particular case, we consider a nonsuperconducting material in $L$, i.e., $\Delta_{L}=0$. The eigenspinors of the KS equations now have the form

$$
\begin{aligned}
\widetilde{\Phi}_{L}^{\tau m}(\mathbf{r}) \equiv & \left(\begin{array}{c}
\widetilde{u}_{L, \uparrow}^{\tau m}(\mathbf{r}) \\
\widetilde{u}_{L, \downarrow}^{T m}(\mathbf{r}) \\
\widetilde{v}_{L, \uparrow}^{\tau m}(\mathbf{r}) \\
\widetilde{v}_{L, \downarrow}^{\tau m}(\mathbf{r})
\end{array}\right)=\left(\begin{array}{c}
\phi_{m, \uparrow}(\mathbf{r}) \\
\phi_{m, \downarrow}(\mathbf{r}) \\
0 \\
0
\end{array}\right) \delta_{\tau, p}+\left(\begin{array}{c}
0 \\
0 \\
\phi_{m, \uparrow}^{*}(\mathbf{r}) \\
\phi_{m, \downarrow}^{*}(\mathbf{r})
\end{array}\right) \delta_{\tau, h} \\
& (\tau=p, h),
\end{aligned}
$$

where the labels $\tau=p$ and $\tau=h$ designate particlelike and holelike KS quasiparticles, respectively. The amplitudes $\phi_{m, \sigma}(\mathbf{r})$ are solutions of an eigenvalue problem analogous to the Bogolubov-de Gennes equations [Eq. 15 in Ref. 7]. For a given $m$, the corresponding eigenvalue $\varepsilon_{m}$ can either be positive or negative and the external potentials are

$$
v_{\sigma}\left(\varepsilon_{v} ; \boldsymbol{x}^{\prime}, \boldsymbol{y}^{\prime}\right)=\int_{L} d^{3} x t\left(\boldsymbol{x}^{\prime}, \boldsymbol{x}\right) \sum_{m} \int_{L} d^{3} y \frac{\phi_{m, \sigma}(\boldsymbol{x}) \phi_{m, \sigma}^{*}(\boldsymbol{y})}{\varepsilon_{\nu}-\varepsilon_{m}} t\left(\boldsymbol{y}, \boldsymbol{y}^{\prime}\right),
$$

$$
S\left(\varepsilon_{\nu} ; \boldsymbol{x}^{\prime}, \boldsymbol{y}^{\prime}\right)=\int_{L} d^{3} x t\left(\boldsymbol{x}^{\prime}, \boldsymbol{x}\right) \sum_{m} \int_{L} d^{3} y \frac{\phi_{m, \uparrow}(\boldsymbol{x}) \phi_{m, \downarrow}^{*}(\boldsymbol{y})}{\varepsilon_{\nu}-\varepsilon_{m}} t\left(\boldsymbol{y}, \boldsymbol{y}^{\prime}\right),
$$

$$
\Delta\left(\varepsilon_{\nu} ; \boldsymbol{x}^{\prime}, \boldsymbol{y}^{\prime}\right)=\Delta_{m}\left(\varepsilon_{\nu} ; \boldsymbol{x}^{\prime}, \boldsymbol{y}^{\prime}\right)=0 \quad(m=-1,0,1) .
$$

This set is analogous to Eqs. (39a)-(39c) and gives substance to the image $^{7}$ of the external contribution to the staggered field in DFT as a proximity effect, an interpretation that places additional emphasis on the analogy between DFT for superconductors and for spin-density waves.

\section{GENERALIZATION: MAGNETIC INTERFACES}

The tunneling Hamiltonian (27) defines an inert barrier, one that merely allows charge transport between regions $L$ and $R$. By contrast, this section examines a magnetically active interface in which spin-orbit coupling or spin-flip scattering from impurities add off-diagonal elements to the tunneling matrix. In special situations, a trivial rotation of the quantization axis may be sufficient to diagonalize that matrix over the entire barrier; in such instances, one expects the barrier to induce spin polarization in both $L$ and $R$.

More generally, however, the spatial dependence of the matrix elements will bar global diagonalization so that instead of simply redefining the quantization axis, the barrier will turn into an inhomogeneous source of spin flips. While the system, constituted by the $L$ and $R$ regions and the interface between them, is subject to global conservation laws, the spin of the electrons in regions $R$ and $L$ is no longer conserved. This section explores the consequences of that rupture. We shall see that while the potentials $v_{\sigma}(\sigma=\uparrow, \downarrow)$ and $S$ are only quantitatively affected, the magnetic barrier breaks the independence between the singlet and the triplet Cooper pairs. A magnetic interface separating a singlet superconductor from a normal metal, for instance, will induce the formation of triplet pairs in the latter.

More specifically, instead of Eq. (27) we consider the following tunneling KS Hamiltonian:

$$
H_{\text {Tmagn }}^{(s)}=\left(\begin{array}{cccc}
\hat{t}_{\uparrow \uparrow} & \hat{t}_{\uparrow \downarrow} & 0 & 0 \\
\hat{t}_{\downarrow \uparrow} & \hat{t}_{\downarrow \downarrow} & 0 & 0 \\
0 & 0 & -\hat{t}_{\uparrow \uparrow}^{*} & -\hat{t}_{\uparrow \downarrow}^{*} \\
0 & 0 & -\hat{t}_{\downarrow \uparrow}^{*} & -\hat{t}_{\downarrow \downarrow}^{*}
\end{array}\right),
$$

and follow the analysis leading from Eq. (27) to Eq. (34), which now takes the form

$$
H_{\text {ext,magn }}\left(\varepsilon_{\nu}\right)=\sum_{\ell} H_{\mathrm{Tmagn}}^{(s)} \widetilde{\Phi}_{L}^{\ell} \frac{1}{\varepsilon_{\nu}-\widetilde{\varepsilon}_{L}^{\varphi}} \widetilde{\Phi}_{L}^{\ell \dagger} H_{\mathrm{Tmagn}}^{(s)} .
$$

From Eqs. (15) and (44), the computation of the righthand side of Eq. (45) is straightforward, which brings Eq. (45) to the form of Eq. (36), with the kernels

$$
\begin{aligned}
v_{\tau}\left(\varepsilon_{\nu} ; \boldsymbol{x}^{\prime}, \boldsymbol{y}^{\prime}\right)= & \int_{L} d^{3} x \sum_{\sigma, \sigma^{\prime}} t_{\tau \sigma}\left(\boldsymbol{x}^{\prime}, \boldsymbol{x}\right) \int_{L} d^{3} y \sum_{\ell} \frac{\widetilde{u}_{L, \sigma}^{\ell}(\boldsymbol{x}) \widetilde{u}_{L, \sigma^{\prime}}^{\ell *}(\boldsymbol{y})}{\varepsilon_{\nu}-\widetilde{\varepsilon}_{L, l}} \\
& \times t_{\sigma^{\prime} \tau}\left(\boldsymbol{y}, \boldsymbol{y}^{\prime}\right), \quad(\tau=\uparrow, \downarrow),
\end{aligned}
$$




$$
\begin{aligned}
S\left(\varepsilon_{\nu} ; \boldsymbol{x}^{\prime}, \boldsymbol{y}^{\prime}\right)= & \int_{L} d^{3} x \sum_{\sigma, \sigma^{\prime}} t_{\uparrow \sigma}\left(\boldsymbol{x}^{\prime}, \boldsymbol{x}\right) \int_{L} d^{3} y \sum_{\ell} \frac{\widetilde{u}_{L, \sigma}^{\ell}(\boldsymbol{x}) \widetilde{u}_{L, \sigma^{\prime}}^{\ell *}(\boldsymbol{y})}{\varepsilon_{\nu}-\widetilde{\varepsilon}_{L, l}} \\
& \times t_{\sigma^{\prime} \downarrow}\left(\boldsymbol{y}, \boldsymbol{y}^{\prime}\right),
\end{aligned}
$$

and, with the notation $\mathcal{P}_{\tau \tau^{\prime}}^{s m}\left(\tau, \tau^{\prime}=\uparrow, \downarrow, \mathcal{S}=0,1\right.$, and $m$ $=-\mathcal{S}, \ldots, \mathcal{S})$ for the spin operator projecting the doublets $\tau$ and $\tau^{\prime}$ onto their singlet $(\mathcal{S}=0)$ or triplet $(\mathcal{S}=1)$ combinations, ${ }^{45}$

$$
\begin{aligned}
\Delta\left(\varepsilon_{\nu} ; \boldsymbol{x}^{\prime}, \boldsymbol{y}^{\prime}\right)= & \frac{1}{2} \mathcal{P}_{\tau \tau^{\prime}}^{00} \int_{L} d^{3} x \sum_{\sigma, \sigma^{\prime}} t_{\tau \sigma}\left(\boldsymbol{x}^{\prime}, \boldsymbol{x}\right) \int_{L} d^{3} y \\
& \times \sum_{\ell} \frac{\widetilde{u}_{L, \sigma}^{\ell}(\boldsymbol{x}) \widetilde{v}_{L, \sigma^{\prime}}^{\ell *}(\boldsymbol{y})}{\varepsilon_{\nu}-\widetilde{\varepsilon}_{L, l}} t_{\sigma^{\prime} \tau^{\prime}}^{*}\left(\boldsymbol{y}, \boldsymbol{y}^{\prime}\right), \\
\Delta_{m}\left(\boldsymbol{\varepsilon}_{\nu} ; \boldsymbol{x}^{\prime}, \boldsymbol{y}^{\prime}\right)= & \frac{1}{2} \mathcal{P}_{\tau \tau^{\prime}}^{1 m} \int_{L} d^{3} x \sum_{\sigma, \sigma^{\prime}} t_{\tau \sigma}\left(\boldsymbol{x}^{\prime}, \boldsymbol{x}\right) \int_{L} d^{3} y \\
& \times \sum_{\ell} \frac{\widetilde{u}_{L, \sigma}^{\ell}(\boldsymbol{x}) \widetilde{v}_{L, \sigma^{\prime}}^{\ell *}(\boldsymbol{y})}{\varepsilon_{\nu}-\widetilde{\varepsilon}_{L, l}} t_{\sigma^{\prime} \tau^{\prime}}^{*}\left(\boldsymbol{y}, \boldsymbol{y}^{\prime}\right) \\
& (m=0, \pm 1) .
\end{aligned}
$$

While the projector $\mathcal{P}_{\tau \tau^{\prime}}^{S m}$ in Eqs. (46c) and (46d) ensures singlet and triplet symmetries for $\Delta$ and $\Delta_{m}$, respectively, the sums over the spin components $\sigma$ and $\sigma^{\prime}$ involve all combinations of the eigenvectors $\widetilde{u}_{L \sigma}^{\ell}$ and $\widetilde{v}_{L \sigma^{\prime}}^{\ell *}$, i.e., contributions from both the singlet and the triplet anomalous densities. The product $\tilde{u}_{L \uparrow}^{\ell}(\boldsymbol{x}) \tilde{v}_{L \uparrow}^{\ell *}(\boldsymbol{y})$, for instance, which Eq. (23d) associates with $\chi_{1}(\boldsymbol{y}, \boldsymbol{x})$, contributes to $\Delta$ and to all three components of $\Delta_{m}$; should a global rotation of the quantization axis diagonalize both $t\left(\boldsymbol{x}^{\prime}, \boldsymbol{x}\right)$ and $t\left(\boldsymbol{y}, \boldsymbol{y}^{\prime}\right)$, its contribution to the singlet potential $\Delta$ would vanish so that the mismatch between the symmetries of the densities and of the KS potentials would be restricted to the $z$ component of $\chi_{m}$ and $\Delta_{m}$. In general, however, in the presence of magnetically inhomogeneous interfaces neither the $z$ component nor the total spin on one side of the interface is conserved.

In particular, if a singlet superconductor in region $L$ is coupled to a normal metal in region $R$, the singlet anomalous density will contribute to the right-hand side of Eq. (46d). We expect, therefore, triplet Cooper pairs to be proximity induced in the normal metal, along with singlet pairs due to the potential $\Delta$.
To conclude this section we note that the coupling between anomalous densities with different symmetries is in line with spin conservation. The off-diagonal terms in our phenomenological Eq. (44) arise from magnetic degrees of freedom in the barrier, which interact with the spins of the electrons in $L$ and $R$. Only the total spin $S^{2}=\left(S_{L}+S_{T}+S_{R}\right)^{2}$, which includes the contribution $\boldsymbol{S}_{T}$ from such degrees of freedom, must be conserved. While a tunneling matrix (44) diagonalizable by a uniform rotation of its spin variables is sufficient to conserve $S_{L}^{2}$ and $S_{R}^{2}$, and while at least in special situations, this condition proves not necessary, ${ }^{46}$ the righthand side of Eq. (44) will in general fail to commute with $S_{R}^{2}$ and hence allow $\mathcal{S} \rightarrow \mathcal{S} \pm 1$ Cooper-pair spin transitions.

\section{CONCLUSIONS}

We have discussed a density-functional formalism describing superconductivity and magnetism, a generalization encompassing DFT for superconductors, ${ }^{6,18}$ and DFT for antiferromagnets ${ }^{7}$ that covers systems with coexisting order parameters. The formalism being particularly practical in descriptions of two quantum liquids coupled by tunneling, we have derived an exact expression relating the proximity field in one region to the eigenfunctions and eigenvalues of the unperturbed $\mathrm{KS}$ equations in the opposite region.

The solution of the self-consistent cycle of equations presented in Sec. III B gives individual access to the singlet and the triplet Cooper-pair densities as well as to the staggered magnetization. This feature of the formalism was explored in Sec. IV B, which demonstrated that the proximity field induced by the singlet (triplet) density affects only the singlet (triplet) pairs and showed that DFT opens attractive perspectives for the study of the competition between order parameters in junctions such as a conventional or unconventional superconductor coupled to a noncollinear antiferromagnet. In Sec. V we considered the special case of a magnetic interface. We found that a singlet superconductor can in this case induce triplet pairing in a normal metal. We hope that our results stimulate further experimental scrutiny of macroscopically inhomogeneous quantum liquids and serve as the basis for an ab initio description of such systems.

\section{ACKNOWLEDGMENTS}

This work received financial support from the FAPESP and CNPq. J.Q. thanks Nikitas Gidopoulos for some useful comments. J.Q. acknowledges financial support from CCLRC (now STFC) in association with St. Catherine's College, Oxford.
${ }^{1}$ W. Kohn, Rev. Mod. Phys. 71, 1253 (1999).

${ }^{2}$ R. M. Dreizler and E. K. U. Gross, Density Functional Theory (Springer, Berlin, 1990).

${ }^{3}$ U. v. Barth and L. Hedin, J. Phys. C 5, 1629 (1972).

${ }^{4}$ O. Gunnarsson and B. I. Lundqvist, Phys. Rev. B 13, 4274 (1976).

${ }^{5}$ R. O. Jones and O. Gunnarsson, Rev. Mod. Phys. 61, 689
(1989).

${ }^{6}$ L. N. Oliveira, E. K. U. Gross, and W. Kohn, Phys. Rev. Lett. 60, 2430 (1988).

${ }^{7}$ K. Capelle and L. N. Oliveira, Phys. Rev. B 61, 15228 (2000).

${ }^{8}$ M. N. Baibich, J. M. Broto, A. Fert, F. Nguyen Van Dau, F. Petroff, P. Etienne, G. Creuzet, A. Friederich, and J. Chazelas, Phys. Rev. Lett. 61, 2472 (1988). 
${ }^{9}$ G. Binasch, P. Grünberg, F. Saurenbach, and W. Zinn, Phys. Rev. B 39, 4828 (1989).

${ }^{10}$ J. Chakhalian et al., Nat. Phys. 2, 244 (2006).

${ }^{11}$ J. Santamaria, Nat. Phys. 2, 229 (2006).

${ }^{12}$ K. M. Lang, V. Madhavan, J. E. Hoffman, E. W. Hudson, H. Eisaki, S. Uchida, and J. C. Davis, Nature (London) 415, 412 (2002).

${ }^{13}$ G. R. Stewart, Rev. Mod. Phys. 56, 755 (1984).

${ }^{14}$ M. Sigrist and K. Ueda, Rev. Mod. Phys. 63, 239 (1991).

${ }^{15}$ T. Ishiguro and K. Yamagij, Organic Superconductors, Springer Series in Solid State Sciences Vol. 88 (Springer-Verlag, Berlin, 1990).

${ }^{16}$ S. Y. A. Moreo and E. Dagotto, Science 283, 2034 (1999).

${ }^{17}$ Our interest here is not in intrinsic aspects of the proximity effect, occurring over length scales in the order of the correlation length, such as the very long range observed in junctions made of different superconductors R. S. Decca, H. D. Drew, E. Osquiguil, B. Maiorov, and J. Guimpel, Phys. Rev. Lett. 85, 3708 (2000); J. Quintanilla, L. N. Oliveira, and K. Capelle, ibid. 90, 089703 (2003); I. Bozovic, G. Logvenov, M. A. J. Verhoeven, P. Caputo, E. Goldobin, and M. R. Beasley, ibid. 93, 157002 (2004). Rather, we are interested in features due to the atomicscale properties of the interface. It is in the description of such features that an ab initio approach based on DFT would be most useful.

${ }^{18}$ K. Capelle and E. K. U. Gross, Int. J. Quantum Chem. 61, 325 (1997).

${ }^{19}$ P. G. de Gennes, in Superconductivity, edited by R. Parks (Dekker, New York, 1969).

${ }^{20}$ P. G. De Gennes, Rev. Mod. Phys. 36, 225 (1964).

${ }^{21}$ J. Bardeen, L. N. Cooper, and J. R. Schrieffer, Phys. Rev. 108, 1175 (1957).

${ }^{22}$ J. Hubbard, Proc. R. Soc. London, Ser. A 276, 238 (1963).

${ }^{23}$ G. M. Eliashberg, Sov. Phys. JETP 11, 696 (1960).

${ }^{24}$ S. Kurth, M. Marques, M. Luders, and E. K. U. Gross, Phys. Rev. Lett. 83, 2628 (1999).

${ }^{25}$ M. A. L. Marques, M. Luders, N. N. Lathiotakis, G. Profeta, A. Floris, L. Fast, A. Continenza, E. K. U. Gross, and S. Massidda, Phys. Rev. B 72, 024546 (2005).

${ }^{26}$ A. Floris, G. Profeta, N. N. Lathiotakis, M. Luders, M. A. L. Marques, C. Franchini, E. K. U. Gross, A. Continenza, and S.
Massidda, Phys. Rev. Lett. 94, 037004 (2005).

${ }^{27}$ M. Luders, M. A. L. Marques, N. N. Lathiotakis, A. Floris, G. Profeta, L. Fast, A. Continenza, S. Massidda, and E. K. U. Gross, Phys. Rev. B 72, 024545 (2005).

${ }^{28}$ G. Profeta, C. Franchini, N. N. Lathiotakis, A. Floris, A. Sanna, M. A. L. Marques, M. Luders, S. Massidda, E. K. U. Gross, and A. Continenza, Phys. Rev. Lett. 96, 047003 (2006).

${ }^{29}$ A. Floris, A. Sanna, S. Massidda, and E. K. U. Gross, Phys. Rev. B 75, 054508 (2007).

${ }^{30}$ A. Sanna, G. Profeta, A. Floris, A. Marini, E. K. U. Gross, and S. Massidda, Phys. Rev. B 75, 020511 (2007).

${ }^{31}$ L. M. Sandratskii, Adv. Phys. 47, 91 (1998).

${ }^{32}$ L. Nordstrom and D. J. Singh, Phys. Rev. Lett. 76, 4420 (1996).

${ }^{33}$ J. Kubler, K. H. Hock, J. Sticht, and A. R. Williams, J. Phys. F: Met. Phys. 18, 469 (1988).

${ }^{34}$ J. Kubler, K. H. Hock, J. Sticht, and A. R. Williams, J. Appl. Phys. 63, 3482 (1988).

${ }^{35}$ The first order correction of the error involved is given in N. I. Gidopoulos, Phys. Rev. B 75, 134408 (2007).

${ }^{36}$ S. Sharma, J. K. Dewhurst, C. Ambrosch-Draxl, S. Kurth, N. Helbig, S. Pittalis, S. Shallcross, L. Nordström, and E. K. U. Gross, Phys. Rev. Lett. 98, 196405 (2007).

${ }^{37}$ K. Capelle and L. N. Oliveira, Europhys. Lett. 49, 376 (2000).

${ }^{38}$ K. Capelle, M. F. Silva, and L. N. Oliveira, J. Magn. Magn. Mater. 226-230, 1017 (2001).

${ }^{39}$ P. W. Anderson, Phys. Rev. 112, 1900 (1958).

${ }^{40}$ P. A. Fedders and P. C. Martin, Phys. Rev. 143, 245 (1966).

${ }^{41}$ C. Herring, in Magnetism, edited by G. T. Rado and H. Suhl (Academic, New York, 1963), Vol. 4.

${ }^{42}$ A. W. Overhauser, Phys. Rev. Lett. 4, 462 (1960).

${ }^{43}$ A. W. Overhauser, Phys. Rev. 128, 1437 (1962).

${ }^{44}$ R. E. Prange, Phys. Rev. 131, 1083 (1963).

${ }^{45}$ For instance, $\quad \mathcal{P}_{\tau \tau^{\prime}}^{00} t_{\tau \sigma}\left(\boldsymbol{x}^{\prime}, \boldsymbol{x}\right) t_{\sigma^{\prime} \tau^{\prime}}\left(\boldsymbol{y}, \boldsymbol{y}^{\prime}\right) \equiv\left[t_{\uparrow \sigma}\left(\boldsymbol{x}^{\prime}, \boldsymbol{x}\right) t_{\sigma^{\prime} \downarrow}\left(\boldsymbol{y}, \boldsymbol{y}^{\prime}\right)\right.$ $\left.-t_{\downarrow \sigma}\left(\boldsymbol{x}^{\prime}, \boldsymbol{x}\right) t_{\sigma^{\prime} \uparrow}\left(\boldsymbol{y}, \boldsymbol{y}^{\prime}\right)\right], \quad$ and $\quad \mathcal{P}_{\tau \tau^{\prime}}^{11} t_{\tau \sigma}\left(\boldsymbol{x}^{\prime}, \boldsymbol{x}\right) t_{\sigma^{\prime} \tau^{\prime}}\left(\boldsymbol{y}, \boldsymbol{y}^{\prime}\right)$ $\equiv t_{\uparrow \sigma}\left(\boldsymbol{x}^{\prime}, \boldsymbol{x}\right) t_{\sigma^{\prime} \uparrow}\left(\boldsymbol{y}, \boldsymbol{y}^{\prime}\right)$.

${ }^{46}$ As an example of a tunneling matrix that cannot be diagonalized by a global rotation and still conserves $S_{L}^{2}$ and $S_{R}^{2}$, consider a matrix diagonalizable by a uniform rotation everywhere except in a region where the barrier is so high that the eigenfunctions $u^{\ell}$ and $v^{\ell}$ vanish. 